\title{
Prior Anticoagulation in Patients with Ischemic Stroke and Atrial Fibrillation
}

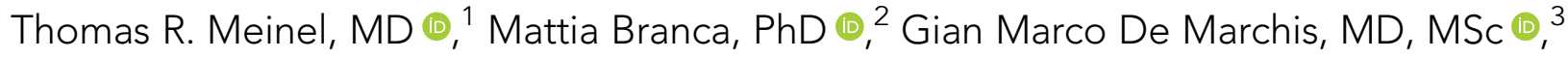 \\ Krassen Nedeltchev, MD, ${ }^{4}$ Timo Kahles, MD, ${ }^{4}$ Leo Bonati, MD, ${ }^{3}$ Marcel Arnold, MD, ${ }^{1}$ \\ Mirjam R. Heldner, MD, MS $\odot,{ }^{1}$ Simon Jung, MD, ${ }^{1}$ Emmanuel Carrera, MD ${ }^{\circ}{ }^{5}$ \\ Elisabeth Dirren, MD, ${ }^{5}$ Patrik Michel, MD, ${ }^{6}$ Davide Strambo, MD, ${ }^{6}$ \\ Carlo W. Cereda, MD $\odot_{1}^{7}$ Giovanni Bianco, MD, ${ }^{7}$ Georg Kägi, MD, ${ }^{8}$ Jochen Vehoff, MD, ${ }^{8}$ \\ Mira Katan, MD, ${ }^{9}$ Manuel Bolognese, MD, ${ }^{10}$ Roland Backhaus, MD, ${ }^{11}$ \\ Stephan Salmen, MD, ${ }^{12}$ Sylvan Albert, MD, ${ }^{13}$ Friedrich Medlin, MD, ${ }^{14}$ \\ Christian Berger, MD, ${ }^{15}$ Ludwig Schelosky, MD, ${ }^{16}$ Susanne Renaud, MD, ${ }^{17}$ \\ Julien Niederhauser, MD, ${ }^{18}$ Christophe Bonvin, MD, MSc, ${ }^{19}$ Michael Schaerer, MD, ${ }^{20}$ \\ Marie-Luise Mono, MD, ${ }^{21}$ Biljana Rodic, MD, ${ }^{22}$ Alexander A. Tarnutzer, MD, ${ }^{23}$ \\ Pasquale Mordasini, MD, ${ }^{24}$ Jan Gralla, MD, ${ }^{24}$ Johannes Kaesmacher, MD, ${ }^{25}$ \\ Stefan Engelter, MD, ${ }^{3,26 \dagger}$ Urs Fischer, MD, MSc, ${ }^{1 \dagger}$ and David J. Seiffge, MD, ${ }^{1+}$ \\ the Investigators of the Swiss Stroke Registry
}

Objective: The aim was to evaluate, in patients with atrial fibrillation (AF) and acute ischemic stroke, the association of prior anticoagulation with vitamin $\mathrm{K}$ antagonists (VKAs) or direct oral anticoagulants (DOACs) with stroke severity, utilization of intravenous thrombolysis (IVT), safety of IVT, and 3-month outcomes.

Methods: This was a cohort study of consecutive patients (2014-2019) on anticoagulation versus those without (controls) with regard to stroke severity, rates of IVT/mechanical thrombectomy, symptomatic intracranial hemorrhage $(\mathrm{sICH})$, and favorable outcome (modified Rankin Scale score $0-2)$ at 3 months.

View this article online at wileyonlinelibrary.com. DOI: 10.1002/ana.25917

Received Jun 3, 2020, and in revised form Sep 23, 2020. Accepted for publication Sep 23, 2020.

Address correspondence to Dr Seiffge, Department of Neurology, Inselspital, Freiburgstrasse 8, CH-3010, Switzerland, E-mail: david.seiffge@insel.ch

${ }^{\dagger}$ Equally contributing senior authors.

Members of the Swiss Stroke Registry are available as an online supplementary file.

From the ${ }^{1}$ Department of Neurology, Inselspital, Bern University Hospital, and University of Bern, Bern, Switzerland; ${ }^{2}$ Clinicial Trials Unit Bern, University of Bern, Bern, Switzerland; ${ }^{3}$ Department of Neurology and Stroke Center, University Hospital Basel and University of Basel, Basel, Switzerland; ${ }^{4}$ Department of Neurology, Kantonsspital Aarau, Aarau, Switzerland; ${ }^{5}$ Department of Neurology, Hôpitaux Universitaires de Genève, Geneva, Switzerland; ${ }^{6}$ Stroke Center, Neurology Service, Lausanne University Hospital, Lausanne, Switzerland; ${ }^{7}$ Stroke Center, Neurocenter of Southern Switzerland, Lugano, Switzerland; ${ }^{8}$ Department of Neurology, Kantonsspital St. Gallen, St. Gallen, Switzerland; ${ }^{9}$ Department of Neurology, University Hospital Zurich, Basel, Switzerland; ${ }^{10}$ Neurocenter, Cantonal Hospital of Lucerne, Lucerne, Switzerland; ${ }^{11}$ Hirslanden Hospital Zurich, Glattpark, Switzerland; ${ }^{12}$ Spitalzentrum Biel,

Biel, Switzerland; ${ }^{13}$ Cantonal Hospital Graubuenden, Chur, Switzerland; ${ }^{14}$ Stroke Unit, Cantonal Hospital Fribourg, Fribourg, Switzerland; ${ }^{15}$ Spital

Sarganserland Grabs, Grabs, Switzerland; ${ }^{16}$ Neurology, Cantonal Hospital Münsterlingen, Münsterlingen, Switzerland; ${ }^{17}$ Neurology, Cantonal Hospital Neuchatel, Neuchâtel, Switzerland; ${ }^{18}$ Stroke Unit, Groupement hospitalier de l'ouest lémanique, Nyon, Switzerland; ${ }^{19}$ Hôpital du Valais, Sion, Switzerland;

${ }^{20}$ Bürgerspital Solothurn, Solothurn, Switzerland; ${ }^{21}$ Stadtspital Waid und Triemli, Zurich, Switzerland; ${ }^{22}$ Cantonal Hospital Winterthur, Winterthur, Switzerland; ${ }^{23}$ Cantonal hospital of Baden, Baden, Switzerland; ${ }^{24}$ Institute of Diagnostic and Interventional Neuroradiology, Inselspital, Bern University Hospital, and University of Bern, Bern, Switzerland; ${ }^{25}$ Department of Neurology, Institute of Diagnostic and Interventional Neuroradiology, Institute of Diagnostic, Interventional and Pediatric Radiology, Inselspital, Bern University Hospital, and University of Bern, Bern, Switzerland; and ${ }^{26}$ Neurology and Neurorehabilitation, University Department of Geriatic Medicine Felix Platter, University of Basel, Basel, Switzerland 
Results: Of 8,179 patients (mean [SD] age, 79.8 [9.6] years; 49\% women), 1,486 (18\%) were on VKA treatment, 1,634 (20\%) on DOAC treatment at stroke onset, and 5,059 controls. Stroke severity was lower in patients on DOACs (median National Institutes of Health Stroke Scale 4, [interquartile range 2-11]) compared with VKA (6, [2-14]) and controls (7, [3-15], $p<0.001$; quantile regression: $\beta-2.1$, 95\% confidence interval [Cl] -2.6 to -1.7$)$. The IVT rate in potentially eligible patients was significantly lower in patients on VKA (156 of 247 [63\%]; adjusted odds ratio [aOR] 0.67; 95\% $\mathrm{Cl} 0.50-0.90$ ) and particularly in patients on DOACs (69 of 464 [15\%]; aOR $0.06 ; 95 \% \mathrm{Cl} 0.05-0.08$ ) compared with controls (1,544 of 2,504 [74\%]). sICH after IVT occurred in 3.6\% (2.6-4.7\%) of controls, 9 of 195 (4.6\%; 1.9-9.2\%; aOR 0.93; $95 \% \mathrm{Cl} 0.46-1.90)$ patients on VKA and 2 of 65 (3.1\%; $0.4-10.8 \%$, aOR $0.56 ; 95 \% \mathrm{Cl} 0.28-1.12)$ of those on DOACs. After adjustments for prognostic confounders, DOAC pretreatment was associated with a favorable 3-month outcome (aOR 1.24; 1.01-1.51).

Interpretation: Prior DOAC therapy in patients with AF was associated with decreased admission stroke severity at onset and a remarkably low rate of IVT. Overall, patients on DOAC might have better functional outcome at 3 months. Further research is needed to overcome potential restrictions for IVT in patients taking DOACs.

ANN NEUROL 2021;89:42-53

$\mathrm{O}$ ral anticoagulation with direct oral anticoagulants (DOACs) or vitamin K antagonists (VKAs) is highly efficacious in the prevention of acute ischemic stroke in patients with atrial fibrillation (AF). ${ }^{1-3}$ Nevertheless, ischemic stroke occurs in $-2 \%$ of patients on anticoagulants per year, ${ }^{4,5}$ and $-10 \%$ of all ischemic stroke patients are already on anticoagulation therapy at stroke onset. ${ }^{6,7}$

Prior therapeutic VKA and DOAC therapy has been associated with reduced stroke severity at onset as measured using the National Institutes of Health Stroke Scale (NIHSS).${ }^{8-11}$ Acute recanalization therapies (intravenous thrombolysis [IVT] and mechanical thrombectomy [MT]) are the cornerstone of acute stroke therapy, improving functional outcome and preventing major disability after stroke. ${ }^{12}$ Prior anticoagulation is a relative contraindication for IVT $^{12}$ and a risk-benefit judgment is usually made on MT according to current American heart association/American stroke association (AHA/ASA) guidelines. The results from observational studies suggest that IVT and MT are safe in selected patients taking VKAs (if INR is $\leq 1.7)^{13,14}$ and DOACs. ${ }^{13,15-19}$ However, prior anticoagulation therapy poses a major challenge in the hyperacute setting owing to the fear of an increased risk of symptomatic intracranial hemorrhage (sICH). In particular, prior DOAC therapy might represent a major barrier to the use of acute recanalization therapies because of uncertainties surrounding the monitoring of drug effects. Therefore, the benefits of prior DOAC therapy on acute stroke characteristics might conversely be canceled out by poorer long-term functional outcomes owing to a reluctance to use acute recanalization therapies.

We aimed to assess the rate of prior treatment with VKAs or DOACs in patients with AF and acute ischemic stroke in a national dataset and determine its association with: (1) stroke severity and the presence of large vessel occlusion (LVO) at onset, (2) use of acute recanalization therapies and incidence of their complications $(\mathrm{sICH})$, and (3) functional outcome at 3 months.

\section{Patients and Methods}

\section{Study Population and Setting}

This is an analysis of data from the Swiss Stroke Registry (SSR), a compulsory prospectively constructed national registry. Since 2014, all consecutive patients hospitalized in stroke units and comprehensive stroke centers (all certified according to national Swiss Stroke Unit and Stroke Center criteria, and in line with those of the European Stroke Organisation $)^{20}$ have been enrolled in the SSR, which is designed for quality control and research in acute stroke management. ${ }^{21,22}$ For the present analysis, we included all consecutive ischemic stroke patients with $\mathrm{AF}$ (AF diagnosed either before or after stroke onset) aged $\geq 18$ years who were admitted from January 2014 to October 2019 to hospitals participating in the SSR. We excluded patients with missing data on prior anticoagulation therapy.

This project was planned, designed, and approved by the SSR steering committee, which is composed of multidisciplinary researchers (neurology, neuroradiology, and neurosurgery) in cooperation with the clinical trials units of Basel (data management) and Bern (data analysis).

\section{Baseline Characteristics}

Local investigators at the participating SSR centers collected standardized and prespecified variables using electronic case report forms. The secured, web-based databank is hosted by the clinical trials unit in Basel. The following variables were used in this analysis: patient demographic characteristics (age, sex, and body mass index), medical history (eg, history of prior ischemic stroke, intracerebral hemorrhage, hypertension, dyslipidemia, diabetes, AF, and smoking), prior anticoagulation therapy on admission (no anticoagulation $=$ controls, $\mathrm{VKA}$, or DOAC), clinical information (NIHSS score, blood glucose, and blood pressure on admission, and international normalized ratio 
[INR] for patients taking VKA), in addition to the diagnostic tests performed (eg, imaging modality, presence of LVO). Information on recanalization treatments included the use of IVT and/or MT plus details on dose, workflow metrics, and mode of application. We collected additional information from every stroke center and stroke unit on: (1) availability of a standard operating procedure for IVT and MT in patients on DOACs, (2) availability and turnaround time (median, estimate) of drug-specific coagulation assays for DOACs plasma levels, ${ }^{23}$ (3) availability and local standard operating procedure for use of drug-specific reversal agents for DOACs (ie, idarucizumab and andexanet alfa) before IVT or MT in patients taking DOACs, and (4) reasons to withhold IVT in patients on DOACs potentially eligible for IVT. ${ }^{24,25}$ A plausibility check was applied, including restrictions for age, NIHSS, blood pressure, body mass index, and symptom onset to hospital time (see data analysis plan in the Online Supplement), and data items were set as missing if they were implausible.

\section{Prior Anticoagulation Therapy}

Patients with prior anticoagulation therapy were defined as those on prescribed anticoagulation therapy at the time of stroke symptom onset. For this analysis, anticoagulation treatments were categorized into three mutually exclusive groups: (1) controls (patients with AF without anticoagulation), (2) VKA, and (3) DOACs (rivaroxaban, dabigatran, apixaban, or edoxaban). For sensitivity analysis, we divided the VKA group into patients with confirmed therapeutic (admission INR >1.7) and nontherapeutic (INR $\leq 1.7$, ie, not within the therapeutic range) drug treatment. Patients with other types of anticoagulation, such as heparins, were also excluded from this analysis, whereas additional prescription of antiplatelet agents had no effect on group allocation.

\section{Follow-Up and Outcomes}

All patients enrolled in the SSR received standardized follow-up assessments by local investigators for in-hospital (sICH according to ECASS-II criteria [an intracranial hemorrhage was defined as symptomatic if the patient had clinical deterioration causing an increase in the NIHSS score of more than or equal to 4 points and if the hemorrhage was likely to be the cause of the clinical deterioration]) and 3-month outcomes (functional outcome using the modified Rankin Scale score [mRS]). All follow-up checks were performed by mRS-certified stroke neurologists or trained research staff during clinic visits, or by structured telephone interviews conducted by mRScertified examiners.
The main outcomes for this analysis were: (1) stroke severity at admission (NIHSS), (2) presence of LVO, (3) rates of IVT and MT, (4) occurrence of symptomatic intracranial hemorrhage (ECASS-II definition), and (5) favorable functional outcome at 3 months (mRS 0-2). See Fig 1 for flow diagram of outcome completeness.

\section{Statistical Analysis}

The statistical analysis followed a predefined statistical analysis plan (T.R.M., M.B., and D.J.S.). We used the median (interquartile range [IQR]) or mean (standard deviation $[\mathrm{SD}]$ ), as appropriate, and percentages, to present the distribution of continuous, ordinal, and categorical variables, respectively. We compared the baseline characteristics across the prior anticoagulation treatment groups using the Pearson $\chi^{2}$ test for categorical variables and the Kruskal-Wallis test for continuous and ordinal variables.

For the primary analysis, we used univariate and multiple regression models to investigate the association between prior anticoagulation therapy and the main outcomes (NIHSS at onset; rates of IVT, MT, and sICH; and favorable outcome at 3 months). Prior anticoagulation was included as an independent variable, with controls (patients with AF without anticoagulation) as the reference group, and prior VKAs and DOACs as the other categorical groups. All analyses were performed on the cohort of ischemic stroke patients with AF unless otherwise indicated.

To assess the association of the NIHSS and presence of LVO at stroke onset with prior anticoagulation therapy, the following covariates were included in a quantile (NIHSS) or binary multiple (LVO) regression model: age, sex, admission glucose, previous ischemic stroke, previous transient ischemic attack, arterial hypertension, diabetes mellitus, dyslipidemia, smoking, and systolic blood pressure.

To analyze the association of prior anticoagulation and acute recanalization therapies (IVT and MT) with the complication (sICH), we restricted the analysis to patients who were potentially eligible for acute recanalization therapies. For questions in potentially IVT-eligible patients, we included patients presenting 0 to 4.5 hours after symptom onset and excluded patients with INR $>1.7$, platelet count $<100 \mathrm{~g} / \mathrm{l}$, admission glucose $<2.7$ or $>22.2 \mathrm{mmol} / \mathrm{l}$, NIHSS $<4$, and patients with a history of intracerebral hemorrhage before the current ischemic stroke onset. For MT, we included patients presenting 0 to 6 hours after symptom onset and with a NIHSS $\geq 6$. We present the rate of IVT, MT, and sICH with $95 \%$ confidence intervals (CI) and relevant procedural metrics (door-to-needle and door-to-groin-puncture time). We performed 


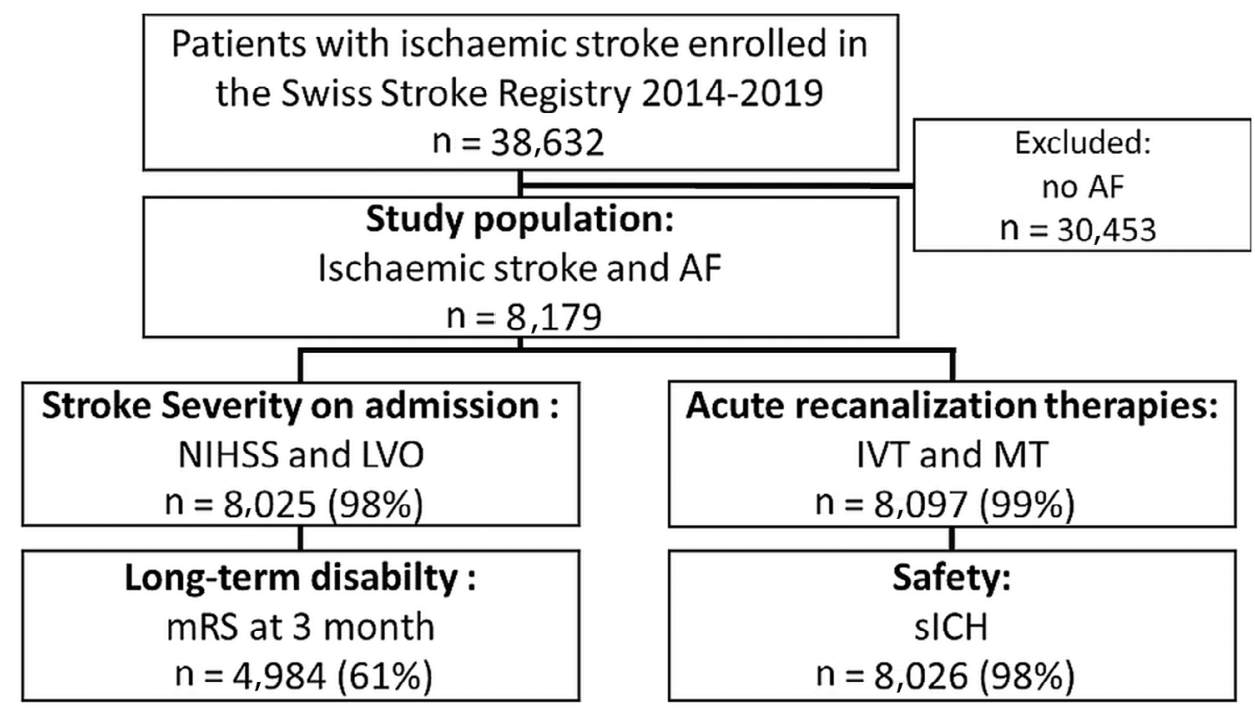

FIGURE 1: Flow diagram of outcome completeness. AF = atrial fibrillation; IVT = intravenous thrombolysis; LVO = large vessel occlusion; $\mathrm{mRS}=$ modified Rankin Scale; MT = mechanical thrombectomy; NIHSS = National Institutes of Health Stroke Scale; sICH = symptomatic intracranial hemorrhage.

univariate comparisons of factors associated with receiving IVT and MT. To assess the association between IVT and prior anticoagulation therapy, we performed univariate comparisons and applied a stepwise multiple regression model to identify factors associated with receiving IVT.

To assess the association between prior anticoagulation and functional outcome at 3 months, we performed two multivariate models. Initially, we adjusted a basic model for prespecified baseline confounders including age, sex, admission NIHSS, and use of IVT and MT. In an additional advanced model, we adjusted for admission glucose ( 1 if $>7.3$ or $<3.7 \mathrm{mmol} / 1 ; 0$ if $3.7-$ $7.3 \mathrm{mmol} / \mathrm{l}$ ), onset to admission time ( 1 if $>3$ hours from symptom onset to admission; 0 if $0-3$ hours), previous ischemic stroke, previous intracerebral hemorrhage, arterial hypertension, diabetes mellitus, and systolic blood pressure ( 1 if $>180$ or $<140 \mathrm{mmHg}$; 0 if $140-180 \mathrm{mmHg}$ ). Patients with missing outcome measures or missing data items were excluded from the multivariable analysis. For the interaction analysis between prior anticoagulation treatment, IVT, and 3-month functional outcome, we included an interaction term of IVT eligibility and anticoagulation in the logistic regression model.

We performed the following post-hoc sensitivity analyses. To determine the relationship between NIHSS and LVO, we used mediation analysis using the Stata gformula function. Furthermore, as we assumed that DOACs protect against severe stroke, a lower admission NIHSS would be a covariate on the pathway of causality between DOAC pretreatment and 3-month outcome; therefore, adjusting for admission NIHSS might constitute an over-adjustment. Therefore, we repeated the multivariate analysis without NIHSS. Furthermore, we included pre-stroke mRS in the model to adjust for prior disability. Finally, we repeated the multivariate models using ordinal shift analysis instead of dichotomized mRS at 3 months.

We calculated (adjusted) odds ratios (aORs) and the corresponding 95\% CI. All statistical analyses were performed using STATA (StataCorp. 2019. Stata Statistical Software: Release 16. College Station, TX: StataCorp LLC). All $p$-values are 2 -sided, with $p<.05$ considered statistically significant, and without adjustments for multiple testing.

\section{Ethics}

The registry and this analysis were both approved by the responsible ethics committee (KEK Bern 2019-01010). In accordance with Swiss law, patients who, after being informed about the collection of their biological data, refused to allow its use for research purposes were excluded from the analysis. This study complies with the Declaration of Helsinki.

\section{Results}

\section{Baseline Characteristics}

Of all 38,632 patients with ischemic stroke admitted to 13 certified Swiss stroke units and 10 stroke centers between January 5, 2014 and October 8, 2019, 8,179 (21.2\%) had pre-existing or newly diagnosed AF (mean [SD] age, 79.8 [9.6] years; 49\% women; median NIHSS 6). Ninety-five of 38,632 patients $(0.25 \%)$ were excluded because of missing information on prior anticoagulation therapy. Among all stroke patients, 2,472 patients (6.4\%) 

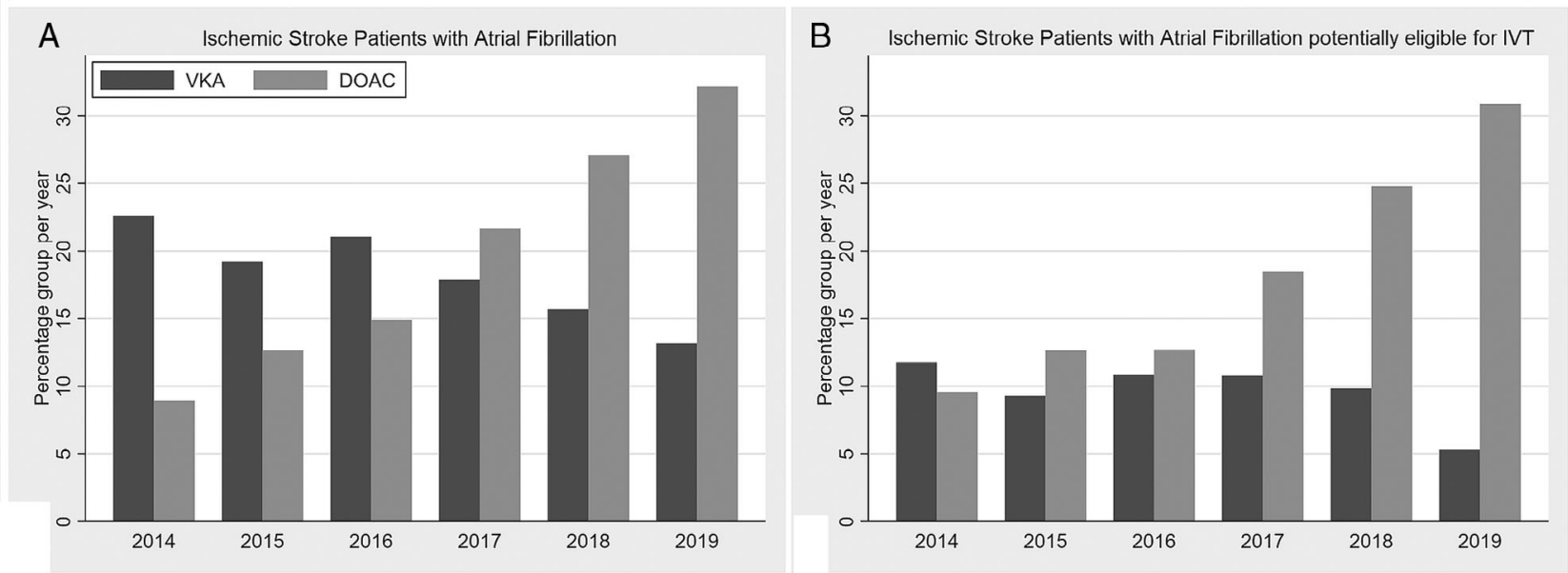

FIGURE 2: Frequency of prior anticoagulation treatment in patients with ischemic stroke. (A) Percentages of patients with atrial fibrillation among all ischemic stroke patients. (B) Percentages of patients with atrial fibrillation within the subgroup of ischemic stroke patients potentially eligible for intravenous thrombolysis. Note the decrease in VKA pretreatment with concomitant rapid increase in DOAC pretreatment in both patient groups. DOACs = direct oral anticoagulants; IVT = intravenous thrombolysis; $\mathrm{VKA}=$ vitamin $\mathrm{K}$ antagonist.

had prior DOAC treatment and 2,368 patients $(6.1 \%)$ prior VKA treatment. Among ischemic stroke patients with AF, 1,486 (18\%) patients had received preceding VKA and $1,634(20 \%)$ preceding DOAC treatment (rivaroxaban 1,040, apixaban 408, dabigatran 99 and edoxaban 87), with decreasing rates of VKA and increasing rates of DOACs over time, whereas 5,059 (62\%) were controls (Fig 2). Of the VKA-treated patients, 609 (41\%) had a subtherapeutic therapy (INR $\leq 1.7$ ). Among the ischemic stroke patients with AF potentially eligible for IVT, 466 (18\%) had prior DOAC treatment and $247(9.5 \%)$ prior VKA treatment, with rapidly increasing numbers of DOAC pretreatment in recent years (see Fig 2).

Ischemic stroke patients with AF showed baseline differences in age, medical history of ischemic stroke, transient ischemic attack, hypertension, diabetes mellitus, dyslipidemia, smoking, symptom onset-to-hospital time, and systolic and diastolic blood pressures (Table 1). Owing to missing data items, $98 \%$ of patients were included in the basic and $82 \%$ in the advanced multiple regression models.

\section{Stroke Severity and Presence of Large Vessel Occlusion at Onset}

Information on stroke severity at onset was available for $98 \%$ of patients $(n=8,025)$. Patients on DOACs had a lower stroke severity score (NIHSS median 4, IQR [2-11]) than patients on VKA (6 [2-14]) and controls (7 [3-15], $p<0.001$; Table 2) in unadjusted analysis. Stroke was less severe in patients on therapeutic VKA (5 [2-12]) compared with those outside the therapeutic range (8 [3-16], $p<0.001)$. After adjustments for confounders, in comparison to controls, stroke was more severe ( $\beta$ indicates the median difference in NIHSS score) in patients with subtherapeutic VKA $(\beta 1.4,95 \%$ CI $0.1-$ 2.6, $p=0.031)$ and less severe in those with therapeutic values of VKA $(\beta-1.8,95 \%$ CI -2.5 to -1.1 , $p<0.001)$ and in patients on DOACs $(\beta-2.1,95 \%$ CI -2.6 to $-1.7, p<0.001)$. When comparing all patients on VKA regardless of INR, stroke was milder $(\beta-0.9$, $95 \% \mathrm{CI}-1.5$ to $-0.3, p=0.004)$ than in controls, but even less severe in patients on DOACs $(\beta-2.1,95 \%$ CI -2.5 to $-1.7, p<0.001)$. LVO was present in $51 \%$ of controls, $44 \%$ of patients on VKA, and $39 \%$ of those on DOACs $(p<0.001)$. After adjustment for confounders, LVO occurred less often in patients on VKAs (aOR 0.81, 95\% CI 0.70-0.93) and DOACs (aOR 0.67, 95\% CI $0.58-0.76)$ than in controls. LVO was also less frequent in patients on DOACs than in those on VKA (aOR 0.82, 95\% CI $0.69-0.99, p=0.038$ ). The post-hoc mediation analysis showed that presence of LVO accounted for $77 \%$ (95\% CI $64-91 \%, p<0.001)$ of NIHSS severity.

\section{Acute Recanalization Therapies: IVT, MT, and $\mathrm{sICH}$}

Information on IVT, MT, and sICH was available for 99\% (IVT and MT) and 98\% (sICH, $\mathrm{n}=8,026)$ of patients, respectively. Among patients potentially eligible for IVT, the rate of IVT was 1,544 of 2,504 (74\%; $95 \%$ CI $72-76 \%)$ in controls, 156 of 247 (63\%; 57-69\%) in patients on VKA (with INR 1.7$)$, and 69 of $464(15 \%$; $12-18 \%)$ in patients on DOACs. Patients on prior anticoagulation therapy had longer door-to-needle times, with 


\begin{tabular}{|c|c|c|c|c|c|c|c|}
\hline Characteristic & $\begin{array}{l}\text { All patients with } \\
\text { AF }(n=8,179)\end{array}$ & $\begin{array}{l}\text { Patients with AF } \\
\text { without } \\
\text { anticoagulation } \\
(\mathrm{n}=5,059)\end{array}$ & $\begin{array}{l}\text { Vitamin } K \text { antago } \\
\text { All }(n=1,486)\end{array}$ & $\begin{array}{l}\text { onists } \\
\text { INR } \\
\leq 1.7(n=609)\end{array}$ & $\begin{array}{l}\text { INR } \\
>1.7(\mathrm{n}=877)\end{array}$ & $\begin{array}{l}\text { DOACs } \\
(\mathrm{n}=1,634)\end{array}$ & $p^{\mathrm{a}}$ \\
\hline Age, yr & $\begin{array}{l}\mathrm{n}=8,179 \\
79.8 \pm 9.6\end{array}$ & $\begin{array}{c}\mathrm{n}=5,059 \\
79.4 \pm 10.1\end{array}$ & $\begin{array}{l}\mathrm{n}=1,486 \\
81.3 \pm 8.2\end{array}$ & $\begin{array}{c}\mathrm{n}=609 \\
82.1 \pm 7.8\end{array}$ & $\begin{array}{c}\mathrm{n}=877 \\
80.7 \pm 8.4\end{array}$ & $\begin{array}{l}\mathrm{n}=1,634 \\
79.8 \pm 8.9\end{array}$ & $<0.001$ \\
\hline Sex, male & $\begin{array}{c}\mathrm{n}=8,173,4207 \\
(51 \%)\end{array}$ & $\begin{array}{c}\mathrm{n}=5,055,2,558 \\
(51 \%)\end{array}$ & $\mathrm{n}={ }_{(52 \%)}^{1,485,766}$ & $\mathrm{n}=\underset{(46 \%)}{608,279}$ & $\begin{array}{c}\mathrm{n}=877,487 \\
(56 \%)\end{array}$ & $\mathrm{n}={ }_{(54 \%)}^{1,633,883}$ & 0.051 \\
\hline $\begin{array}{l}\text { Body mass index, } \\
\mathrm{kg} / \mathrm{m}^{2}\end{array}$ & $\begin{array}{l}\mathrm{n}=5,745 \\
25.8 \pm 4.6\end{array}$ & $\begin{array}{l}\mathrm{n}=3,637 \\
25.8 \pm 4.6\end{array}$ & $\begin{array}{l}\mathrm{n}=1,029 \\
26.0 \pm 4.6\end{array}$ & $\begin{array}{c}\mathrm{n}=402 \\
25.7 \pm 4.3\end{array}$ & $\begin{array}{c}\mathrm{n}=627 \\
26.3 \pm 4.7\end{array}$ & $\begin{array}{l}\mathrm{n}=1,079 \\
25.8 \pm 4.7\end{array}$ & 0.202 \\
\hline $\begin{array}{l}\text { Previous ischemic } \\
\text { stroke, yes }\end{array}$ & $\begin{array}{c}\mathrm{n}=8,100,1784 \\
(22 \%)\end{array}$ & $\begin{array}{c}\mathrm{n}=4,998,847 \\
(17 \%)\end{array}$ & $\mathrm{n}=\underset{(27 \%)}{1,473,401}$ & $\mathrm{n}=\underset{(27 \%)}{602,163}$ & $\begin{array}{c}\mathrm{n}= \\
(27 \%)\end{array}$ & $\begin{aligned} \mathrm{n}= & 1,629,536 \\
(33 \%) & \end{aligned}$ & $<0.001$ \\
\hline $\begin{array}{l}\text { Previous transient } \\
\text { ischemic attack, yes }\end{array}$ & $\begin{array}{c}\mathrm{n}=8,093,578 \\
(7 \%)\end{array}$ & $\begin{array}{c}\mathrm{n}=4,998,281 \\
(6 \%)\end{array}$ & $\mathrm{n}=\begin{array}{l}1,470,146 \\
(10 \%)\end{array}$ & $\mathrm{n}=\underset{(8 \%)}{599,46}$ & $\begin{array}{c}\mathrm{n}=871,100 \\
(11 \%)\end{array}$ & $\begin{array}{c}\mathrm{n}=1,625,151 \\
(9 \%)\end{array}$ & $<0.001$ \\
\hline $\begin{array}{l}\text { Previous intracerebral } \\
\text { hemorrhage, yes }\end{array}$ & $\mathrm{n}=\frac{8,101,195}{(2 \%)}$ & $\mathrm{n}=5,000,131$ & $\begin{aligned} \mathrm{n}= & 1,474,31 \\
& (2 \%)\end{aligned}$ & $\mathrm{n}=\underset{(2 \%)}{603,12}$ & $\mathrm{n}=\underset{(2 \%)}{871,19}$ & $\mathrm{n}=\underset{(2 \%)}{1,627,33}$ & 0.281 \\
\hline Hypertension, yes & $\begin{array}{c}\mathrm{n}=8,132,6,823 \\
(84 \%)\end{array}$ & $\begin{array}{c}\mathrm{n}=5,018,4,092 \\
(82 \%)\end{array}$ & $\begin{array}{c}\mathrm{n}=1,482 \\
1,306(88 \%)\end{array}$ & $\begin{array}{c}\mathrm{n}=605,545 \\
(90 \%)\end{array}$ & $\begin{array}{c}\mathrm{n}=877,761 \\
(87 \%)\end{array}$ & $\begin{array}{c}\mathrm{n}=1,632 \\
1,425(87 \%)\end{array}$ & $<0.001$ \\
\hline Diabetes mellitus, yes & $\begin{array}{c}\mathrm{n}=8,127,1,868 \\
(23 \%)\end{array}$ & $\begin{array}{c}\mathrm{n}=5,016,1,060 \\
(21 \%)\end{array}$ & $\mathrm{n}=\underset{(27 \%)}{1,480,406}$ & $\mathrm{n}=\underset{(27 \%)}{604,165}$ & $\begin{array}{c}\mathrm{n}=876,241 \\
(28 \%)\end{array}$ & $\mathrm{n}=\underset{(25 \%)}{1,631,402}$ & $<0.001$ \\
\hline Dyslipidemia, yes & $\begin{array}{c}\mathrm{n}=8,079,5,001 \\
(62 \%)\end{array}$ & $\begin{array}{c}\mathrm{n}=4,990,2,975 \\
(60 \%)\end{array}$ & $\mathrm{n}=\mathrm{f}_{(66 \%)}^{1,469,964}$ & $\begin{array}{c}\mathrm{n}=\underset{(64 \%)}{600,384} \\
\text { (64) }\end{array}$ & $\begin{array}{c}\mathrm{n}=869,580 \\
(67 \%)\end{array}$ & $\begin{array}{c}\mathrm{n}=1,620 \\
1,062(66 \%)\end{array}$ & $<0.001$ \\
\hline Smoker, yes & $\mathrm{n}=\frac{8,020,978}{(12 \%)}$ & $\mathrm{n}=\underset{(13 \%)}{4,932,649}$ & $\begin{aligned} \mathrm{n}= & 1,469,140 \\
(10 \%) & \end{aligned}$ & $\mathrm{n}=\underset{(8 \%)}{602,49}$ & $\begin{array}{c}\mathrm{n}=867,91 \\
(10 \%)\end{array}$ & $\begin{aligned} \mathrm{n}= & 1,619,189 \\
& (12 \%)\end{aligned}$ & 0.001 \\
\hline $\begin{array}{l}\text { Onset to hospital time, } \\
\text { min }\end{array}$ & $\begin{array}{c}\mathrm{n}=7,670 \\
591.8 \pm 1331.1\end{array}$ & $\begin{array}{c}\mathrm{n}=4,754 \\
554.7 \pm 1289.7\end{array}$ & $\begin{array}{c}\mathrm{n}=1,397 \\
603.0 \pm 1328.5\end{array}$ & $\begin{array}{c}\mathrm{n}=578 \\
400.6 \pm 1302.8\end{array}$ & $\begin{array}{c}\mathrm{n}=819 \\
745.8 \pm 1328.6\end{array}$ & $\begin{array}{c}\mathrm{n}=1,519 \\
697.8 \pm 1451.0\end{array}$ & 0.001 \\
\hline INR value & & & $\begin{array}{c}\mathrm{n}=1450,1.9 \\
(1.5 ; 2.4)\end{array}$ & $\begin{array}{c}\mathrm{n}=609,1.4 \\
(1.2 ; 1.6)\end{array}$ & $\begin{array}{c}\mathrm{n}=841,2.3 \\
(2.0 ; 2.8)\end{array}$ & & \\
\hline Glucose, mmol/l & $\begin{array}{l}\mathrm{n}=6,720 \\
7.3 \pm 2.9\end{array}$ & $\begin{array}{l}\mathrm{n}=4,159 \\
7.3 \pm 2.9\end{array}$ & $\begin{array}{l}\mathrm{n}=1,221 \\
7.3 \pm 2.5\end{array}$ & $\begin{array}{c}\mathrm{n}=483 \\
7.2 \pm 2.4\end{array}$ & $\begin{array}{l}\mathrm{n}=738 \\
7.3 \pm 2.6\end{array}$ & $\begin{array}{l}\mathrm{n}=1,340 \\
7.2 \pm 3.0\end{array}$ & 0.743 \\
\hline $\begin{array}{l}\text { Blood pressure, } \\
\text { systolic, } \mathrm{mmHg}\end{array}$ & $\begin{array}{c}\mathrm{n}=7,850 \\
155.5 \pm 27.7\end{array}$ & $\begin{array}{c}\mathrm{n}=4,841 \\
156.2 \pm 27.9\end{array}$ & $\begin{array}{c}\mathrm{n}=1,434 \\
154.2 \pm 27.1\end{array}$ & $\begin{array}{c}\mathrm{n}=586 \\
154.4 \pm 27.4\end{array}$ & $\begin{array}{c}\mathrm{n}=848 \\
154.0 \pm 26.9\end{array}$ & $\begin{array}{c}\mathrm{n}=1,575 \\
154.2 \pm 27.9\end{array}$ & 0.007 \\
\hline $\begin{array}{l}\text { Blood pressure, } \\
\text { diastolic, } \mathrm{mmHg}\end{array}$ & $\begin{array}{c}\mathrm{n}=7,846 \\
85.4 \pm 18.2\end{array}$ & $\begin{array}{c}\mathrm{n}=4,837 \\
85.9 \pm 18.3\end{array}$ & $\begin{array}{c}\mathrm{n}=1,436 \\
85.1 \pm 18.2\end{array}$ & $\begin{array}{c}\mathrm{n}=586 \\
84.8 \pm 18.2\end{array}$ & $\begin{array}{c}\mathrm{n}=850 \\
85.2 \pm 18.2\end{array}$ & $\begin{array}{c}\mathrm{n}=1,573 \\
84.4 \pm 17.9\end{array}$ & 0.018 \\
\hline
\end{tabular}

a time delay of 15 minutes in patients on VKA and 14 minutes in patients on DOACs in comparison to controls $(p<0.001$; Table 2). Among patients on DOAC therapy receiving IVT, $59(86 \%)$ were treated at a stroke center and $10(15 \%)$ at a stroke unit. sICH after IVT occurred in 49 of $1,363(3.6 \% ; 2.6-4.7 \%)$ controls, 9 of 195 (4.6\%; 1.9-9.2\%) patients on VKA with INR $\leq 1.7$, and 2 of 65 (3.1\%; 0.4-10.8\%, $p=0.796)$ patients on DOACs (Table 2). After adjustment for confounders, there was no difference in the occurrence of sICH between patients on VKAs with INR $\leq 1.7$ (aOR 1.6, 0.8-3.1) or on DOACs (aOR $0.6(0.2-1.6)$ and controls. Among patients potentially eligible for MT (presenting $\leq 6$ hours after symptom onset, with NIHSS $\geq 6$ ), the rate of MT was $43 \%$ (95\% CI 40-45\%) in controls, $42 \%$ $(36-49 \%)$ in patients on VKAs with INR $\leq 1.7$, and $43 \%$ $(38-48 \%)$ in those on DOACs $(p=0.951)$. There was also no significant time delay in symptom onset-to-groinpuncture in patients on VKAs (mean 371 minutes) and DOACs (375 minutes) compared with controls (364 minutes, $p=0.685$ ).

Among all 23 certified stroke units and stroke centers, $21(91 \%)$ had a standard operating procedure to select patients on DOACs for IVT and MT that included prespecified selection criteria (ie, time since last intake, drug-specific coagulation assay, and nonspecific coagulation assays). Most centers (18 of $21,86 \%$ ) used drugspecific coagulation assays (ie, calibrated anti-Xa activity for apixaban, edoxaban, or rivaroxaban; or thrombin time for dabigatran), which apply previously published cut-off values for IVT. ${ }^{15,26}$ Among centers making drug-specific coagulation measurements, the estimated turnaround time for these assays (ie, time from blood sampling to first results available) was $<60$ minutes for 16 of 18 (89\%) and 


\begin{tabular}{|c|c|c|c|c|c|c|}
\hline Outcome & Control & VKA, all & VKA (INR $\leq 1.7)$ & VKA (INR >1.7) & DOACs & $p^{\mathrm{a}}$ \\
\hline \multicolumn{7}{|l|}{ Stroke severity at onset } \\
\hline $\begin{array}{l}\text { NIHSS, median } \\
\text { (IQR) }\end{array}$ & $\begin{array}{c}7.0(3.0 ; 15.0) \\
\mathrm{n}=4,964\end{array}$ & $\begin{array}{c}6.0(2.0 ; 14.0) \\
\mathrm{n}=1,458\end{array}$ & $\begin{array}{c}8.0(3.0 ; 16.0) \\
\mathrm{n}=604\end{array}$ & $\begin{array}{c}5.0(2.0 ; 12.0) \\
\mathrm{n}=854\end{array}$ & $\begin{array}{c}4.0(2.0 ; 11.0) \\
\mathrm{n}=1,603\end{array}$ & $<0.001$ \\
\hline$\beta, 95 \% \mathrm{CI}$ & 1 [Reference] & $\begin{array}{c}-0.91(-1.53 \text { to } \\
-0.29)\end{array}$ & $1.38(0.13-2.63)$ & $\begin{array}{c}-1.78(-2.45 \text { to } \\
-1.10)\end{array}$ & $\begin{array}{c}-2.11(-2.54 \text { to } \\
-1.67)\end{array}$ & \\
\hline \multicolumn{7}{|c|}{ Acute recanalization therapies ${ }^{b}$} \\
\hline Thrombolysis & & & & I & & \\
\hline No./Total No. & $1,544 / 2,504$ & & $156 / 247$ & & $69 / 464$ & $<0.001$ \\
\hline Percentage (95\% CI) & $73.7 \%(71.6-75.7)$ & & $\begin{array}{c}63.2 \% \\
(56.8-69.2)\end{array}$ & & $14.9 \%(11.8-18.4)$ & \\
\hline aOR $(95 \% \mathrm{CI})$ & 1 [Reference] & & $0.67(0.50-0.90)$ & & $0.06(0.05-0.08)$ & \\
\hline $\begin{array}{l}\text { Admission to IVT } \\
\text { start, min }\end{array}$ & $\mathrm{n}=1,500$ & & $\mathrm{n}=164$ & & $\mathrm{n}=72$ & $<0.001$ \\
\hline Mean \pm SD & $34.2 \pm 48.1$ & & $49.2 \pm 41.4$ & & $48.1 \pm 48.5$ & \\
\hline $\begin{array}{l}\text { Change from } \\
\text { control, min }\end{array}$ & - & & 15 & & 13.9 & \\
\hline sICH after IVT & & & & & & 0.796 \\
\hline Percentage (95\% CI) & $3.6 \%(2.6-4.7)$ & & $4.6 \%(1.9-9.2)$ & & $3.1 \%(0.4-10.8)$ & \\
\hline $\mathrm{aOR}(95 \% \mathrm{CI})^{\mathrm{b}}$ & 1 [Reference] & & $1.56(0.78-3.14)$ & & $0.59(0.22-1.61)$ & \\
\hline $\begin{array}{l}\text { Mechanical } \\
\text { thrombectomy }\end{array}$ & & & & & & 0.951 \\
\hline No./Total No. & $726 / 1,707$ & & $168 / 304$ & & $170 / 394$ & \\
\hline Percentage $(95 \% \mathrm{CI})$ & $42.5 \%(40.2-44.9)$ & & $\begin{array}{c}41.9 \% \\
(35.5-48.5)\end{array}$ & & $43.1 \%(38.2-48.2)$ & \\
\hline sICH after MT & $3.3 \%(2.5-4.2)$ & & $4.3 \%(2.1-7.8)$ & & $1.3 \%(0.4-3.0)$ & 0.060 \\
\hline \multicolumn{7}{|c|}{ Functional outcome at $3 \mathrm{mo}$} \\
\hline \multicolumn{7}{|l|}{$\mathrm{mRS} 0-2$ at $3 \mathrm{mo}$} \\
\hline No./Total No. & $1,753 / 3,113$ & $477 / 879$ & $171 / 348$ & $306 / 531$ & $597 / 992$ & 0.004 \\
\hline Percentage (95\% CI) & $56.3 \%(54.5-58.1)$ & $54.3 \%(50.9-57.6)$ & $\begin{array}{c}49.1 \% \\
(43.8-54.5)\end{array}$ & $\begin{array}{c}57.6 \% \\
(53.3-61.9)\end{array}$ & $60.2 \%(57.1-63.2)$ & \\
\hline aOR $(95 \%$ CI $)$ basic $^{c}$ & 1 [Reference] & $1.07(0.90-1.28)$ & $0.99(0.77-1.28)$ & $1.13(0.91-1.41)$ & $1.13(0.95-1.34)$ & \\
\hline $\begin{array}{l}\text { aOR }(95 \% \mathrm{CI}) \\
\text { advanced }^{\mathrm{d}}\end{array}$ & 1 [Reference] & $1.13(0.93-1.38)$ & $1.01(0.76-1.35)$ & $1.22(0.96-1.56)$ & $1.24(1.01-1.51)$ & \\
\hline
\end{tabular}

${ }^{\mathrm{a}}$ Univariate comparison between controls, all VKAs (or VKA with INR $\leq 1.7$ for acute recanalization therapy outcomes) and DOACs.

${ }^{\mathrm{b}}$ Among potentially eligible patients: for IVT: 0 to 4.5 hours after symptom onset and excluding patients with INR $>1.7$, platelet count $<100 \mathrm{~g} / \mathrm{l}$, admission glucose $<2.7 \mathrm{mmol} / \mathrm{l}$ or $>22.2 \mathrm{mmol} / \mathrm{l}$, NIHSS $<4$ and patients with a history of intracerebral hemorrhage before ischemic stroke onset. For MT: 0 to 6 hours after symptom onset and NIHSS $\geq 6$.

${ }^{\circ}$ The basic functional outcome model adjusts for baseline demographic and clinical variables before the index stroke event, including age, sex, admission NIHSS, use of IVT and endovascular treatment. Excludes 40\% of records (3,195 with missing outcome and 102 missing adjustment values).

${ }^{\mathrm{d}}$ The advanced functional outcome model adjusts additionally for admission glucose, onset to admission time, previous ischemic stroke, previous intracerebral hemorrhage, arterial hypertension, diabetes mellitus and systolic blood pressure. Excludes $50 \%$ of records (3,195 with missing outcome and 933 missing adjustment).

$\mathrm{aOR}=$ adjusted odds ratio; $\mathrm{CI}=$ confidence interval; $\mathrm{DOACs}=$ direct oral anticoagulants; $\mathrm{INR}=$ international normalized ratio; $\mathrm{IQR}=$ interquartile range; IVT = intravenous thrombolysis; $\mathrm{mRS}=$ modified Rankin Scale; MT = mechanical thrombectomy; NIHSS = National Institutes of Health Stroke Scale; sICH ECASS II = symptomatic intracranial hemorrhage according to the European Co-operative Acute Stroke Study-II definition; $\mathrm{VKA}=$ vitamin $\mathrm{K}$ antagonist.

$<30$ minutes for 5 of $18(28 \%)$ centers. Eighteen of 23 centers $(78 \%)$ reported that their internal guidelines include evaluation of the use of specific reversal agents (ie, idarucizumab for dabigatran, or andexanet alfa for apixaban or rivaroxaban) in selected patients on DOACs before IVT (or MT). Andexanet alfa was not available in Switzerland during the study period (2014-2019). Only a few patients in Switzerland take dabigatran (thus the 


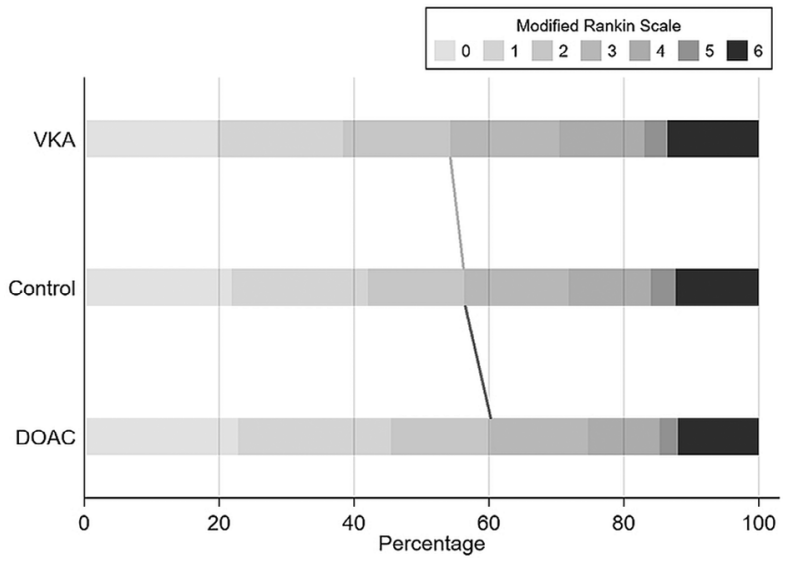

FIGURE 3: Functional outcome at 3 months according to prior anticoagulation treatment in patients with ischemic stroke. The dark gray line indicates a significant association of DOACs (aOR 1.24, 95\% Cl 1.01-1.51), but not VKA (light gray line, aOR $1.13,95 \% \mathrm{Cl} 0.93-1.38)$ with good functional outcome (mRS 0-2). aOR = adjusted odds ratio; $\mathrm{Cl}=$ confidence interval; DOACs = direct oral anticoagulants; $\mathrm{mRS}=$ modified Rankin Scale; VKA = vitamin $\mathrm{K}$ antagonist.

absolut number of patients on dabigatran presenting with stroke in Switzerland is low), and no center reported the use of any reversal agent before IVT or MT.

The main reasons for withholding intravenous thrombolysis in potentially eligible patients on DOACs are presented in Table 3.

\section{Functional Outcome at 3 Months}

Information on the functional outcome at 3 months (mRS) was available for $61 \%$ of patients $(\mathrm{n}=4,984)$, with no differences in availability between the groups $(p=0.249)$. Patients on DOACs $(60 \%)$ had the highest unadjusted rates of favorable outcome at 3 months in comparison to control patients (56\%) and patients on VKA (54\%, $p=0.004$; Fig 3). After adjustment for basic confounders, no significant association of VKA (aOR $1.07,95 \%$ CI $0.90-1.28$ ) or DOACs (aOR $1.13,95 \%$ CI 0.95-1.34) with favorable outcome at 3 months was found. After including additional confounders in the advanced model, there was a significant association of DOACs (aOR 1.24, 95\% CI 1.01-1.51) but not VKAs (aOR 1.13, 95\% CI 0.93-1.38) with favorable outcome. No significant interaction of IVT eligibility with DOACs $(p=0.850)$ or VKA $(p=0.835)$ was seen regarding functional outcome.

Post-hoc sensitivity analyses revealed that the results of the main analysis were consistent if NIHSS was omitted from the model (DOACs: basic model, aOR 1.20, 95\% CI 1.03-1.41, $p=0.022$; advanced model, aOR $1.34,95 \%$ CI 1.12-1.61, $p=0.002)$ or if pre-stroke mRS was included (DOACs: basic model, aOR 1.31, 95\% CI
1.06-1.61, $p=0.011$; advanced model, aOR 1.40, 95\% CI 1.11-1.77, $p=0.004)$. In a model using ordinal shift analysis instead of dichotomized $\mathrm{mRS}$, prior DOAC treatment was associated with lower mRS at 3 months in the advanced model (aOR 0.85, 95\% CI 0.73-0.99, $p=0.041$ ) but not in the basic model (OR 0.93, 95\% CI $0.81-1.06, p=0.257$ ).

\section{Discussion}

The main findings of this large, nationwide registry-based study of ischemic stroke patients with $\mathrm{AF}$ and anticoagulation, in comparison to patients with $\mathrm{AF}$ without anticoagulation (controls) were as follows: First, anticoagulation therapy before stroke onset was common (13\%) among all-cause stroke patients. Second, among ischemic stroke patients with $\mathrm{AF}$, the rate of prior anticoagulation was high (38\%), representing a major challenge among those patients potentially eligible for IVT. The number of patients on DOACs was increasing rapidly throughout the study period. Third, prior DOAC therapy was associated with decreased stroke severity, reduced rates of LVO, and better functional outcome at 3 months when compared with controls. Fourth, although there seem to be no significant restrictions in the use of MT, DOAC pretreatment represented a major barrier to initiation of IVT: patients on DOACs had a 5-fold lower rate of IVT than controls. Fifth, IVT in selected patients on DOACs seemed safe when compared with patients on subtherapeutic VKA or controls.

Previous estimates indicated that $-1 \%$ of patients eligible for IVT will be on a DOAC. ${ }^{27}$ However, the number of patients being prescribed DOACs has increased dramatically in recent years. ${ }^{28}$ Not only have they become the first-line treatment for stroke prevention in patients with non-valvular AF, but they are also prescribed for an increasing number of additional indications. ${ }^{29}$ In accordance with previous studies, we found that 1 in 8 of all stroke patients had been on anticoagulation therapy. However, in the subgroup of AF patients who are potentially eligible for IVT, 1 in 4 patients had prior anticoagulation treatment. This finding, together with the rapidly increasing numbers of patients on DOACs, underlines the importance of establishing safe and reliable management guidelines for recanalization treatments in this population.

Consistent with our hypothesis, the rate of IVT was markedly lower in patients on DOACs than in controls, reflecting the uncertainty felt by clinicians about whether to apply IVT in this clinical situation. Most Swiss stroke units and stroke centers follow a dedicated standard operating procedure to guide treatment decisions. These use 


\section{TABLE 3. Reasons to Withhold Intravenous Thrombolysis in DOAC Patients and Possible Solutions}

Reason to Withhold IVT

DOAC plasma levels too high

Frequency (\%)

Possible Solution to Overcome This

Stroke-related reasons with unclear risk-

benefit (ie, low NIHSS, almost $4.5 \mathrm{~h}$

after onset, infarct demarcation or

regressive symptoms)

DOAC measurement not available

Other patient-related reasons (ie, palliative care decided on admission, frailty, etc.)

Turnaround for DOAC level too long

Direct mechanical thrombectomy preferred

Uncertainity about cut-off values

Surrogate coagulation too high

Other contraindication for IVT

Certain compliance regardless of drug levels

Other suspected diagnosis on admission
At present, it is not known whether patients who have therapeutic DOAC plasma levels have an increased risk of symptomatic intracranial hemorrhage after thrombolyis compared with patients without anticoagulation. International collaboration to gather cases of erroneously thrombolysed patients despite high drug levels.

Establish not only safety, but also efficacy of DOAC reversal for IVT, ideally by a randomized controlled trial.

Not DOAC specific 5 3 3
11

1 Not DOAC specific

\section{Not DOAC specific}

Distribute DOAC test capacities in hospitals providing IVT, especially in smaller hospitals and during non-office hours

Establish rapid and reliable DOAC tests, ideally point of care

Ongoing trials will clarify whether IVT has additional benefits, but according to first trials this seems reasonable

Establish reliable cut-offs of DOAC plasma levels, provided there is an increased risk of symptomatic intracranial hemorrhage in patients on DOACs

Given that correlation of unspecific tests with plasma levels is not sufficient, there is a need to provide capacity to test for specific drug levels and to ascertain compliance reliably

None

See DOAC plasma levels too high

2

1
Not DOAC specific

DOAC $=$ direct oral anticoagulants; IVT $=$ intravenous thrombolysis; NIHSS = National Institutes of Health Stroke Scale. 
drug-specific coagulation assays (in addition to time since last intake) as recommended by recent guidelines and expert opinion. ${ }^{26,30,31}$ Despite these efforts, the rate of IVT was $>5$ times lower in patients on DOACs than in controls.

The most frequent reason for withholding IVT was a high DOAC plasma level, despite the fact that earlier research found that among patients on rivaroxaban, the plasma level was low in most patients at the time of admission. ${ }^{32}$ It seems appropriate to withhold IVT in those patients (primum non nocere), but it is unclear whether IVT genuinely increases the risk of sICH in such patients. Conclusive observational data of erroneously thrombolyzed patients or the use of reversal agents (see below) prior to IVT could overcome this barrier. The second most common reason to withhold IVT was scenarios in which the risk-benefit estimation was deemed uncertain owing to low stroke severity, regressive symptoms, late presentation, or imaging demarcation of the ischemia. For such scenarios, it would also be of utmost importance to clinicians to know whether they should factor therapeutic DOAC therapy into this equation. Another relevant factor was availability and cut-off values for DOAC specific coagulation assays, which require allocation of testing capacities and identification of reliable cut-off values.

Reassuringly, there was no signal towards an increased risk of sICH after application of IVT in patients on DOACs in comparison to controls or patients on VKA (INR $\leq 1.7$ ). This is in line with published reports that found no difference in the occurrence of sICH after IVT in patients on DOACs in comparison to patients without anticoagulation. ${ }^{19,33,34}$ However, the relative safety of IVT in the setting of DOAC pretreatment probably reflects cautious patient selection, because 80 to $90 \%$ of patients on DOACs presenting within the thrombolysis time window are not given IVT. ${ }^{18,35}$

The use of a drug-specific reversal agent before IVT in patients on DOACs has emerged for dabigatran, using idarucizumab. ${ }^{24,25,36}$ Although most Swiss centers have considered this approach, owing to the low market share of dabigatran in Switzerland, no Swiss hospital has reported its use. Andexanet alfa was not available in Switzerland during the study period, and studies are needed to determine whether its use before IVT is safe and effective. However, as shown by our study, there seems to be a large proportion of patients who are potentially eligible for this approach.

Previous studies have shown a reduction in stroke severity in patients on therapeutic VKA $\mathrm{VK}^{8,37,38}$ and $\mathrm{DOAC}^{9}$ therapy, but did not report any long-term outcomes or explanations for this finding. Our results expand these findings with almost identically reduced values for
NIHSS in the anticoagulation categories. Importantly, our data might provide a plausible pathophysiological explanation, because DOAC pretreatment was associated with lower rates of LVO, which usually results in large, severe strokes. Furthermore, our data indicate that the association of stroke severity, when compared with controls, might even be more pronounced in patients on DOACs $(\sim 2$ points less on the NIHSS) than in those on therapeutic VKA $(-1$ point less on the NIHSS). However, the temporal relationship between AF diagnosis and stroke was not evident within the registry, and selection bias for anticoagulation indication might have affected this observation. Other potentially causal factors, such as heart rate control, cardiac output, and adjunctive treatments, might modify stroke severity directly or by influencing thrombus size. Ideally, a pooled analysis of recurrent stroke events in randomized controlled trials could clarify whether DOACs truly reduce stroke severity by lowering the rate of LVO in comparison to VKA.

Our study is the first to report 3-month outcomes, and our adjusted estimates indicate that DOAC pretreatment might be associated with favorable functional outcome at 3 months. However, given the limitations discussed below, further observational studies are necessary to confirm that this favorable association is real, because a randomized controlled trial on pretreatment is impossible.

Finally, we did not find any significant influence of the lower IVT rate among patients on DOACs on the functional outcome at 3 months. However, this finding has to be interpreted with caution because interaction analyses are known to be underpowered. ${ }^{39}$ Interestingly, rates of MT did not differ among patients with prior DOACs, VKAs, and controls. Although the lower rate of IVT among patients on DOACs seems to have no impact on overall outcomes at a population level, we cannot exclude the possibility that withholding IVT for patients on DOACs might have a significant influence on outcome at an individual patient level.

\section{Strengths and Limitations}

Our study has several limitations. First, although data were collected prospectively and independently, the statistical analysis was retrospective and thus prone to bias. Second, the standard operating procedure to select patients on DOACs for IVT differed among centers. Third, although we report data from a large, national dataset, the study was underpowered to show a difference in 3-month outcomes among patients treated with IVT, and we cannot exclude the possibility that the lower rate of IVT in patients on DOACs results in poor outcomes at an individual patient level. Fourth, data completeness was good to excellent for most outcomes, including NIHSS (98\%), 
IVT/MT (99\%), and sICH (98\%), but there was a significant amount of missing data for 3-month outcomes (61\%). Selection bias, residual and unmeasured confounding, and attrition bias might therefore have influenced the results regarding functional outcome of this study. Fifth, thrombin time, an unspecific coagulation assay that might help to guide treatment decisions in patients on dabigatran, was not collected as part of the Swiss stroke registry.

Nevertheless, our study has several strengths. We used data from a national registry enrolling consecutive patients in a prospectively constructed database with predefined variables, which should limit selection bias. Also, we included data from regional stroke units and from tertiary academic and non-academic centers, which means that our findings should be generalizable.

\section{Summary and Conclusions}

One in 8 ischemic stroke patients and 1 in 4 ischemic stroke patients potentially eligible for IVT had prior anticoagulation treatment. Prior DOAC therapy was associated with decreased stroke severity, lower rates of LVO, and a better functional outcome at 3 months when compared with controls. DOAC pretreatment represents a barrier to IVT initiation, although IVT seemed safe in selected patients.

\section{Acknowledgment}

This study was supported by the Bangerter-Rhyner Foundation $(0317 / 2019)$ to TRM.

We thank Susan Kaplan for proofreading the manuscript.

\section{Author Contributions}

T.R.M., M.B., S.T.E., U.F., M.A., L.B., P.M., C.W.C., P.M., E.C., F.M., G.K., K.N., and D.J.S. contributed to the conception and design of the study. T.R.M., M.B., and D.J.S. contributed to the acquisition and analysis of data. G.M.D.M., D.S., T.K., L.B., G.K., P.M., M.H., S.J., E.D., G.B., J.V., M.K., M.B., R.B., S.S., S.A., C.B., C.B., M.S., L.S., S.R., J.N., M.L.M., B.R., A.T., J.K., and J.G. contributed to drafting the text and preparing the figures. L.B. is the Coordinator of the Swiss Stroke Registry

\section{Potential Conflicts of Interest}

The following companies manufacture drugs involved in this study: Bayer (BY; rivaroxaban), Boehringer Ingelheim (BI; dabigatran), Pfizer/Bristol Meyer Squibb (PB; apixaban), and Daiichi Sankyo (DS; edoxaban). M.A.: personal fees, BY, DS, and PB. P.Mic.: research grants, the ERISTA program (PB). M.R.H.: personal fees, BY. L.B.: consultancy or advisory board fees or speaker's honoraria and travel grants, BY and PB. M.K.: research funding, DS. C.B.: nonfinancial support, BI, PB, and BY. M.Bol.: nonfinancial support, BY. C.C.: personal fees, BY, BI, and PB. D.S.: other, PB. G.M.D.M.: personal fees, BY and PB. K.N.: personal fees, BY and PB. All other authors: nothing to report.

\section{Data Availability}

Anonymized data will be shared upon reasonable request from any qualified investigator after clearance by the local ethics committee.

\section{References}

1. Ruff CT, Giugliano RP, Braunwald $E$, et al. Comparison of the efficacy and safety of new oral anticoagulants with warfarin in patients with atrial fibrillation: a meta-analysis of randomised trials. Lancet 2014; 383:955-962. https://doi.org/10.1016/S0140-6736(13)62343-0.

2. Salazar CA, del Aguila D, Cordova EG. Direct thrombin inhibitors versus vitamin $\mathrm{K}$ antagonists for preventing cerebral or systemic embolism in people with non-valvular atrial fibrillation. Cochrane Database Syst Rev 2014;2014:CD009893. https://doi.org/10.1002/ 14651858.CD009893.pub2.

3. Saxena R, Koudstaal PJ. Anticoagulants versus antiplatelet therapy for preventing stroke in patients with nonrheumatic atrial fibrillation and a history of stroke or transient ischemic attack. Cochrane Database Syst Rev 2004;18:CD000187. https://doi.org/10.1002/ 14651858.cd000187.pub2.

4. Almutairi AR, Zhou L, Gellad WF, et al. Effectiveness and safety of non-vitamin $\mathrm{K}$ antagonist oral anticoagulants for atrial fibrillation and venous thromboembolism: a systematic review and meta-analyses. Clin Ther 2017;39:1456-1478.e36. https://doi.org/10.1016/j. clinthera.2017.05.358.

5. Miyamoto S, Ikeda T, Ogawa S, et al. Clinical risk factors of thromboembolic and major bleeding events for patients with atrial fibrillation treated with rivaroxaban in Japan. J Stroke Cerebrovasc Dis 2020;29: 1-9. https://doi.org/10.1016/j.jstrokecerebrovasdis.2019.104584.

6. Auer E, Frey S, Kaesmacher J, et al. Stroke severity in patients with preceding direct oral anticoagulant therapy as compared to vitamin K antagonists. J Neurol 2019;266:2263-2272. https://doi.org/10. 1007/s00415-019-09412-y.

7. Rizos T, Horstmann S, Jenetzky $E$, et al. Oral anticoagulants - a frequent challenge for the emergency management of acute ischemic stroke. Cerebrovasc Dis 2012;34:411-418. https://doi.org/10.1159/ 000343655

8. Xian $Y, O^{\prime}$ Brien EC, Liang $L$, et al. Association of preceding antithrombotic treatment with acute ischemic stroke severity and inhospital outcomes among patients with atrial fibrillation. JAMA 2017;317:1057-1067. https://doi.org/10.1001/jama.2017.1371.

9. Macha K, Marsch A, Siedler G, et al. Cerebral ischemia in patients on direct oral anticoagulants: plasma levels are associated with stroke severity. Stroke 2019;50:873-879. https://doi.org/10.1161/ STROKEAHA.118.023877

10. Hylek EM, Go AS, Chang $Y$, et al. Effect of intensity of oral anticoagulation on stroke severity and mortality in atrial fibrillation. $\mathrm{N}$ Engl J Med 2003;349:1019-1026. https://doi.org/10.1056/ NEJMoa022913.

11. Yi X, Lin J, Han Z, et al. Preceding antithrombotic treatment is associated with acute ischemic stroke severity and functional outcome at 90 days among patients with atrial fibrillation. J Stroke Cerebrovasc 
Dis 2019;28:2003-2010. https://doi.org/10.1016/j.jstrokecerebro vasdis.2019.03.028.

12. Powers WJ, Rabinstein AA, Ackerson T, et al. Guidelines for the early management of patients with acute ischemic stroke: 2019 update to the 2018 guidelines for the early management of acute ischemic stroke a guideline for healthcare professionals from the American Heart Association/American Stroke Association. Stroke 2019;50: E344-E418. https://doi.org/10.1161/STR.0000000000000211.

13. Xian $Y$, Federspiel JJ, Hernandez AF, et al. Use of intravenous recombinant tissue plasminogen activator in patients with acute ischemic stroke who take non-vitamin $\mathrm{K}$ antagonist oral anticoagulants before stroke. Circulation 2017;135:1024-1035. https://doi.org/ 10.1161/CIRCULATIONAHA.116.023940.

14. Mazya MV, Lees KR, Markus R, et al. Safety of intravenous thrombolysis for ischemic stroke in patients treated with warfarin. Ann Neurol 2013;74:266-274. https://doi.org/10.1002/ana.23924.

15. Seiffge DJ, Traenka C, Polymeris AA, et al. Intravenous thrombolysis in patients with stroke taking rivaroxaban using drug specific plasma levels: experience with a standard operation procedure in clinical practice. J Stroke 2017;19:347-355. https://doi.org/10.5853/jos. 2017.00395

16. Purrucker JC, Wolf $M$, Haas $K$, et al. Safety of endovascular thrombectomy in patients receiving non-vitamin $\mathrm{K}$ antagonist oral anticoagulants. Stroke 2016;47:1127-1130. https://doi.org/10.1161/ STROKEAHA.116.012684.

17. Meinel TR, Kniepert JU, Seiffge DJ, et al. Endovascular stroke treatment and risk of intracranial hemorrhage in anticoagulated patients. Stroke 2020;51:892-898. https://doi.org/10.1161/STROKEAHA.119. 026606.

18. Seiffge DJ, Hooff RJ, Nolte $\mathrm{CH}$, et al. Recanalization therapies in acute ischemic stroke patients impact of prior treatment with novel oral anticoagulants on bleeding complications and outcome a pilot study. Circulation 2015;132:1261-1269. https://doi.org/10.1161/ CIRCULATIONAHA.115.015484.

19. Shahjouei S, Tsivgoulis G, Goyal N, et al. Safety of intravenous thrombolysis among patients taking direct oral anticoagulants: a systematic review and meta-analysis. Stroke 2020;51:533-541. https:// doi.org/10.1161/STROKEAHA.119.026426.

20. Waje-Andreassen U, Nabavi DG, Engelter ST, et al. European stroke organisation certification of stroke units and stroke centres. Eur Stroke J 2018;3:220-226. https://doi.org/10.1177/ 2396987318778971

21. Manno C, Disanto G, Bianco G, et al. Outcome of endovascular therapy in stroke with large vessel occlusion and mild symptoms. Neurology 2019;93:E1618-E1626. https://doi.org/10.1212/WNL. 0000000000008362

22. Bonati L, Baumgartner RW, Bonvin C, et al. Ein Werkzeug für die Qualitätssicherung und Forschung. Swiss Med Forum 2016;16: 168-169. https://doi.org/10.4414/smf.2016.02576.

23. Seiffge DJ, Traenka C, Polymeris A, et al. Feasibility of rapid measurement of rivaroxaban plasma levels in patients with acute stroke. J Thromb Thrombolysis 2017;43:112-116. https://doi.org/10.1007/ s11239-016-1431-7.

24. Barber PA, Wu TY, Ranta A. Stroke reperfusion therapy following dabigatran reversal with idarucizumab in a national cohort. Neurology 2020;94:e1968-e1972.
25. Dias FA, Pontes-Neto OM, Seiffge DJ. Idarucizumab before reperfusion therapy in stroke patients on dabigatran. Neurology 2020;94: 811-812.

26. Touzé E, Gruel Y, Gouin-Thibault I, et al. Intravenous thrombolysis for acute ischaemic stroke in patients on direct oral anticoagulants. Eur J Neurol 2018;25:747-752. https://doi.org/10.1111/ene.13582.

27. Pfeilschifter W, Farahmand D, Niemann D, et al. Estimating the quantitative demand of NOAC antidote doses on stroke units. Cerebrovasc Dis 2016;42:415-420. https://doi.org/10.1159/ 000447952.

28. Jung YH, Kim YD, Kim J, et al. Initial stroke severity in patients with atrial fibrillation according to antithrombotic therapy before ischemic stroke. Stroke 2020;51:2733-2741. https://doi.org/10.1161/ STROKEAHA.120.030138.

29. Kennedy C, Ni Choitir C, Clarke S, et al. Direct oral anticoagulants uptake and an oral anticoagulation paradox. $\mathrm{Br} \mathrm{J}$ Clin Pharmacol 2020;86:392-397. https://doi.org/10.1111/bcp.14171.

30. Seiffge DJ, Polymeris AA, Fladt J, et al. Management of patients with stroke treated with direct oral anticoagulants. J Neurol 2018; 265:3022-3033. https://doi.org/10.1007/s00415-018-9061-y.

31. Steiner $T$, Böhm M, Dichgans $M$, et al. Recommendations for the emergency management of complications associated with the new direct oral anticoagulants (DOACs), apixaban, dabigatran and rivaroxaban. Clin Res Cardiol 2013;102:399-412. https://doi.org/10. 1007/s00392-013-0560-7.

32. Seiffge DJ, Kägi G, Michel P, et al. Rivaroxaban plasma levels in acute ischemic stroke and intracerebral hemorrhage. Ann Neurol 2018;83:451-459. https://doi.org/10.1002/ana.25165.

33. Tsivgoulis G, Safouris A. Intravenous thrombolysis in acute ischemic stroke patients pretreated with non-vitamin $\mathrm{K}$ antagonist oral anticoagulants. Stroke 2017;48:2031-2033. https://doi.org/10.1161/ STROKEAHA.117.017206.

34. Shahjouei S, Tsivgoulis G, Bavarsad Shahripour R, et al. Safety of intravenous thrombolysis among stroke patients taking new oral anticoagulants - case series and systematic review of reported cases. J Stroke Cerebrovasc Dis 2015;24:2685-2693. https://doi.org/10. 1016/j.jstrokecerebrovasdis.2015.07.021.

35. Purrucker JC, Haas K, Rizos T, et al. Coagulation testing in acute ischemic stroke patients taking non-vitamin $\mathrm{K}$ antagonist oral anticoagulants. Stroke 2017;48:152-158. https://doi.org/10.1161/ STROKEAHA.116.014963.

36. Beharry J, Waters MJ, Drew R, et al. Dabigatran reversal before intravenous tenecteplase in acute ischemic stroke. Stroke 2020;51: 1616-1619. https://doi.org/10.1161/STROKEAHA.119.028327.

37. Ay $H$, Arsava EM, Gungor $L$, et al. Admission international normalized ratio and acute infarct volume in ischemic stroke. Ann Neurol 2008;64:499-506. https://doi.org/10.1002/ana.21456

38. Wakita M, Yasaka M, Minematsu K, Yamaguchi T. Effects of anticoagulation on infarct size and clinical outcome in acute cardioembolic stroke. Angiology 2002;53:551-556. https://doi.org/10. 1177/000331970205300508

39. Brankovic M, Kardys I, Steyerberg EW, et al. Understanding of interaction (subgroup) analysis in clinical trials. Eur J Clin Invest 2019;49: e13145. https://doi.org/10.1111/eci.13145. 